\title{
High Field MicroMRI Velocimetric Measurement of Quantitative Local Flow Curves
}

Tatiana Nikolaeva, Frank J. Vergeldt, Raquel Serial, Joshua A. Dijksman, Paul Venema, Adrian Voda, John van Duynhoven,* and Henk Van As

Cite This: Anal. Chem. 2020, 92, 4193-4200

Read Online

\section{ACCESS | Lill Metrics \& More | 回 Article Recommendations ｜（） Supporting Information}

ABSTRACT: Performing rheo-microMRI velocimetry at a high magnetic field with strong pulsed field gradients has clear advantages in terms of (chemical) sensitivity and resolution in velocities, time, and space. To benefit from these advantages, some artifacts need to be minimized. Significant sources of such artifacts are chemical shift dispersion due to the high magnetic field, eddy currents caused by the pulsed magnetic field gradients, and possible mechanical instabilities in concentric cylinder (CC) rheo-cells. These, in

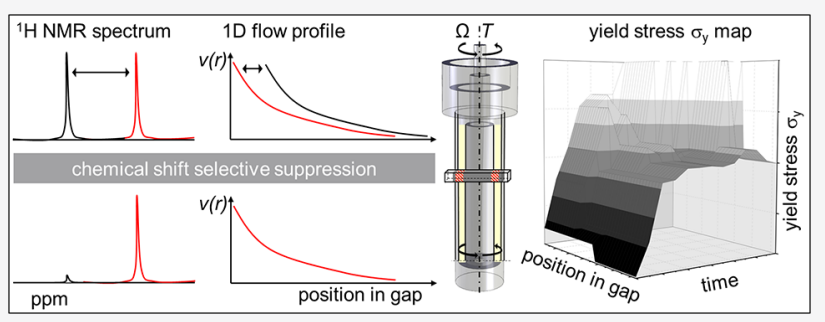
particular, hamper quantitative assessment of spatially resolved velocity profiles needed to construct local flow curves (LFCs) in CC geometries with millimeter gap sizes. A major improvement was achieved by chemical shift selective suppression of signals that are spectroscopically different from the signal of interest. By also accounting for imperfections in pulsed field gradients, LFCs were obtained that were virtually free of artifacts. The approach to obtain quantitative LFCs in millimeter gap CC rheo-MRI cells was validated for Newtonian and simple yield stress fluids, which both showed quantitative agreement between local and global flow curves. No systematic effects of gap size and rotational velocity on the viscosity of a Newtonian fluid and yield stress of a complex fluid could be observed. The acquisition of LFCs during heterogeneous and transient flow of fat crystal dispersion demonstrated that local constitutive laws can be assessed by rheo-microMRI at a high magnetic field in a noninvasive, quantitative, and real-time manner.

$\mathrm{M}$ any colloidal dispersions express their intriguing flow properties in a nonlinear relationship between shear stress and rate. This non-Newtonian flow behavior can be observed for various food products, body fluids, and materials with designed functionalities. ${ }^{1-3}$ The nonlinear relationship between shear stress $\sigma$ and rate $\dot{\gamma}$ can be summarized in socalled flow curves $\sigma(\dot{\gamma}){ }^{4,5}$ These are used to define constitutive laws for flowing materials and classification of non-Newtonian behavior. $^{4-10}$ Well-known classes are shear-thickening or -thinning materials, for which viscosity, respectively, increases or decreases under shear. A peculiar case is presented by yield stress fluids, which behave as elastic solids, when the applied stress is small and as flowing fluids once a critical stress is exceeded. Macroscopic or global flow curves are typically measured by conventional rotational rheometers, ${ }^{4,5,8,11}$ which vary shear stress as a function of shear rate or vice versa. The flow behavior of some materials is such that only wall-derived data are not sufficient to characterize their flow behavior due to heterogeneous flow and/or transient behavior. There are many example fluids to illustrate this, in particular for colloidal suspensions in transient yield stress flow regimes. ${ }^{12-17}$

This limitation can be overcome by rheo-MRI velocimetry which can assess spatially resolved velocities of complex fluids with microscopic resolution. ${ }^{18-27}$ In a label-free, real-time, and noninvasive manner, rheo-MRI can thus uniquely visualize regions governed by different constitutive laws. When rheo-
MRI is performed in a concentric cylinder (CC) or Couette geometry with a known spatial stress distribution $\sigma(\mathrm{r})$ over the gap between the cylinders, ${ }^{28}$ the determination of the spatial distribution of the shear rates $\dot{\gamma}(r)$ opens up the possibility to deduce the constitutive law in the form of a so-called local flow curve (LFC) $\sigma(\dot{\gamma}(r)) .{ }^{17,29,30}$ A main advantage of obtaining LFCs based on rheo-MRI velocimetry is that they can be obtained in a noninvasive real-time manner, which allows monitoring of transient changes in non-Newtonian flow behavior under shear stress. Furthermore, heterogeneous flow in the form of shear banding, ${ }^{22,23,31-33}$ wall slip, ${ }^{18,30}$ and shear-induced migration ${ }^{17,34}$ can be recognized, which facilitates the identification of the underlying colloidal mechanisms.

Currently, rheo-MRI based LFCs are typically obtained using a CC geometry with centimeter-sized gaps, mounted in wide-bore low-field magnets $(0.5 \mathrm{~T})$, which compromises sensitivity and temporal resolution. ${ }^{10,29,30}$ Such systems are

Received: July 16, 2019

Accepted: February 13, 2020

Published: February 13, 2020 
typically equipped with low magnetic field gradients $(0.05 \mathrm{~T} / \mathrm{m})$ and therefore are limited in providing high spatial resolution as well as in capturing small amplitude velocities. MRI velocimetry at a high magnetic field strength $\mathrm{B}_{0}(7 \mathrm{~T})$ with strong magnetic field gradients (typically of the order of $1 \mathrm{~T} / \mathrm{m}$ ) can offer significant improvements in performance. $^{21,25,27,35,36}$ The use of a higher $B_{0}$ field enhances sensitivity and temporal resolution. In addition, the strong gradients allow for the acquisition of profiles of a large dynamic range in rotational velocities with spatial resolution down to tens of micrometers. Therefore, we have coined the implementation at high $\mathrm{B}_{0}$ fields and strong amplitude gradients as rheo-microMRI. The benefits of rheo-microMRI, however, come at the expense of losing robustness toward experimental artifacts. ${ }^{37,38}$

The goal of this work is to show how to recognize and overcome these artifacts when applied to complex colloidal dispersions. Given the chemical complexity of such systems, we can expect multiple signals in the NMR spectrum, which can lead to artifacts due to chemical shift dispersion at high $\mathrm{B}_{0}$ fields. Several of these artifacts are well known and have been addressed in the MRI literature before, also during flow measurements. ${ }^{37,39,40}$ However, the chemical shift artifacts have not been discussed so far in relation to spatially resolved velocimetric measurements performed in a rheo-microMRI setup with millimeter gap-sized CC cells and with strong magnetic field gradients. In addition, we discuss the implications of using strong pulsed field gradients during velocimetric measurements at low rotational velocities, as well as the effects of mechanical instabilities that become noticeable when highly resolved velocity profiles are acquired over a millimeter gap-sized CC cell. The suppression of these artifacts is a prerequisite for obtaining quantitative LFCs. The validation of the LFCs obtained in the millimeter gap-sized CC cell is shown for both Newtonian and non-Newtonian yield stress fluids. The real-time and noninvasive measurements of LFCs by rheo-microMRI for monitoring structure formation will be demonstrated for a micronized fat crystal dispersion which undergoes localized transient structural rearrangements under shear stress.

\section{MATERIALS AND METHODS}

Materials. A commercial olive oil was used as a sample to discuss chemical shift artifacts that can occur during rheomicroMRI experiments. Silicone oil standards of different viscosities (500 and $5000 \mathrm{mPa} s$ at $25{ }^{\circ} \mathrm{C}$ ) (AMETEK Brookfield) were used as Newtonian fluids. A commercial hair gel was used, where Carbopol was the main ingredient responsible for its yield stress behavior. Due to the virtual absence of thixotropy, the hair gel was considered as a simple yield stress fluid. Micronized fat crystal dispersions were used as a model for materials with transient yield stress properties. A powder of micronized fat crystals (MFCs) was dispersed in sunflower oil at a concentration of $10 \mathrm{w} / \mathrm{w} \%$, the full procedure can be found in ref 15. Fat crystal dispersions were obtained under combined intense mixing and vacuum at $20{ }^{\circ} \mathrm{C}$ during $2 \mathrm{~h}$ at constant speed. The fat dispersions were immediately frozen after preparation, stored at $-20{ }^{\circ} \mathrm{C}$, and warmed to room temperature before rheo-microMRI and rheology measurements.

High Field Rheo-microMRI. Rheo-microMRI experiments were conducted on a Bruker Avance III spectrometer, in combination with a commercial rheo-NMR accessory. A vertically wide bore $(89 \mathrm{~mm})$ superconducting magnet with a magnetic field strength $B_{0}=7 \mathrm{~T}$, was used, corresponding to a resonance frequency of $300 \mathrm{MHz}$ for ${ }^{1} \mathrm{H}$. Excitation and detection of the ${ }^{1} \mathrm{H}$ signal was performed with a bird-cage $\mathrm{rf}$ coil with an inner diameter of $25 \mathrm{~mm}$. In the standard microimaging gradient system Micro 2.5 (Bruker), gradients up to $G_{\max }=0.987 \mathrm{~T} / \mathrm{m}$ were available along all three axes. $1 \mathrm{D}$ velocity profiles were measured with a slice thickness of 1 or 2 $\mathrm{mm}$ in two dimensions by a pulsed gradient spin echo (PGSE) sequence implemented for that purpose (mic_SEFLOW in ParaVision version 5.1) with Gaussian rf pulses of duration $p_{90}=1 \mathrm{~ms}$ and $p_{180}=0.59 \mathrm{~ms}$. The velocity-encoding gradient pulses had a duration of $\delta=1 \mathrm{~ms}$, and the observation time was $\Delta=20$ or $13.1 \mathrm{~ms}$. The frequency-encoding read gradient provided a FOV $=25 \mathrm{~mm}$ over 512 pixels, such that the spatial resolution was $\Delta x=48.8 \mu \mathrm{m}$. The time needed to obtain a single velocity profile within the gap was $3 \mathrm{~min} 12 \mathrm{~s}$ or $1 \mathrm{~min}$ $36 \mathrm{~s}$ for, respectively, a number of scans of NS $=64$ or 32 . The velocity profiles were recorded with an echo time of $\mathrm{TE}=$ $17.5 \mathrm{~ms}(\Delta=13.1 \mathrm{~ms})$ or $24.4 \mathrm{~ms}(\Delta=20 \mathrm{~ms})$ and a repetition time $\mathrm{TR}=1.5 \mathrm{~s}$.

Flow experiments were performed with concentric cylinder CC (also called Couette) geometries made from PEEK (Figure 1 ). Both outer and inner cylinders had serrated walls with a

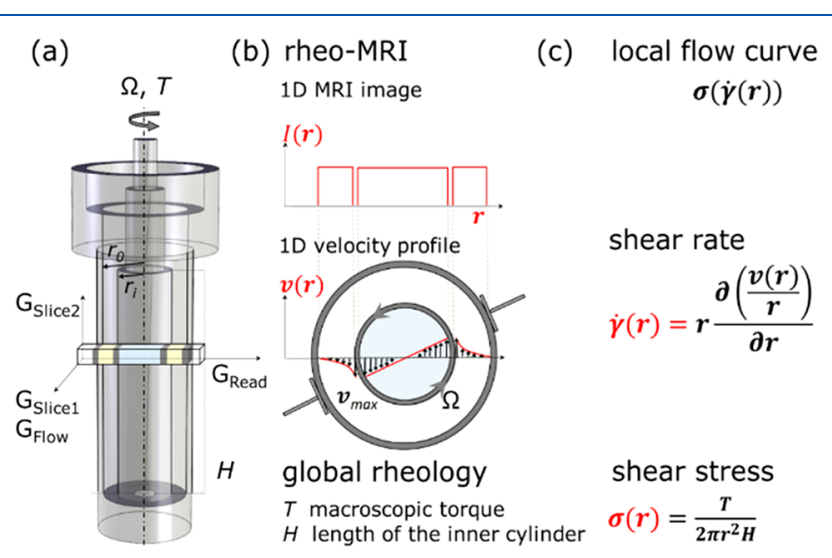

Figure 1. (a) Sketch of the rheo-microMRI concentric cylinder (CC) or Couette cell with a static outer cylinder of radius $r_{\mathrm{o}}$, a rotating inner cylinder of radius $r_{\mathrm{i}}$, and height $H$, rotating with velocity $\Omega$. A column is selected by conventional slice selection with two orthogonal gradients $G_{\text {Slice1 }}$ and $G_{\text {Slice2. }} G_{\text {Flow }}$ and $G_{\text {Read }}$ indicate the directions of flow encoding and read gradients, respectively, which were used to obtain flow as a function of position $r$. (b) Rheo-microMRI provides a spatially resolved $1 \mathrm{D}$ intensity profile $I(r)$ and velocity profile $v(r)$ with a $48.8 \mu \mathrm{m}$ resolution. The velocity profile $v(r)$ is shown as a sketch overlaid on the CC top view, with maximum velocities $\left(v_{\max }\right)$ close to the rotating inner cylinder and zero velocities close to the static outer cylinder. The inner rotating cylinder is hollow and can be filled with a reference fluid (blue). Global rheology provides macroscopic torque values $T$ as a function of time and rotational velocity $\Omega$. (c) Equations are used to calculate local shear rates $\dot{\gamma}(r)$ from rheo-microMRI velocimetric measurements and shear stresses $\sigma(r)$ from global rheology torque measurements in order to construct a local flow curve (LFC) $\sigma(\dot{\gamma}(r))$.

checkered grid with a depth of $100 \mu \mathrm{m}$ and a width/height of approximately $0.5-1 \mathrm{~mm}$ to prevent wall slip. The outer cylinder had a radius of $r_{\mathrm{o}}=11 \mathrm{~mm}$ suited to the $\mathrm{rf}$ insert used in the MicWB40 microMRI probehead. Three different inner cylinders of the same length, $58 \mathrm{~mm}$, were used in order to perform experiments in CC cells with $1,2.5$, and $4 \mathrm{~mm}$ gap 
sizes. The inner rotating cylinders were hollow and filled with a reference fluid. Rheo-microMRI velocity profiles, respectively, comprised of 20,51, and 81 pixels across the CC gap with (48.8 $\mu \mathrm{m}$ resolution). The stability of the ${ }^{1} \mathrm{H}$ NMR signal was monitored in order to ensure that no significant temperature variation occurred during the performed experiments in the rheo-microMRI CC cells. Standard deviations of the velocity values were less than $10 \%$ and were obtained by repeating the measurements 3 to 10 times depending on the type of samples and experimental conditions.

Data Processing. All calculations and corrections of rheomicroMRI velocity profiles, as well as the determination of local shear rates and stresses, were performed in MatlabR2015b (MathWorks). A Savitzky-Golay (SG) FIR smoothing filter (available in Matlab) was used to obtain the first derivative of the velocity data in order to calculate the shear rate variations as a function of position in the gap (Figure $1(c))$. For all experiments, a first-order polynomial fit was used with a window length set to 5 to 7 points, depending on the gap size. LFC data points corresponding to the first 2-4 pixels near the walls $(98-195 \mu \mathrm{m})$ could not be provided, due to the use of the SG filter.

An estimation of apparent yield stress $\sigma_{\mathrm{y}}$ values was done through a fit of the whole LFC or its parts with a power law function presenting the Herschel-Bulkley model. Some of the obtained LFCs could not be fitted by a single power law function over the whole gap due to banding and were therefore pragmatically fitted by two or three power law functions with different parameters, each describing the constitutive law for a band in the gap. ANOVA tests were performed in Excel.

Rheology Measurements. Rheological measurements were performed in parallel to rheo-microMRI experiments. They were conducted on a conventional Modular Compact Rheometer 301 (MCR301, Anton Paar). Global flow curves and macroscopic torque measurements were done in a homebuilt CC cell made from PEEK with exactly the same dimensions and serrated pattern as the rheo-microMRI CC cell (Figure 1) with $1,2.5$, and $4 \mathrm{~mm}$ gap sizes. In comparison to the rheo-microMRI, the inner cylinders were solid in the conventional rheological setup. Before every measurement we verified the rheometer and the home-built CC cell performance with a standard motor adjustment and an air check as provided by the Rheoplus software. The macroscopic torque $T$ measurements were performed using the same conditions and protocols as for rheo-microMRI velocity measurements. The macroscopic torque $T$ measurements were collected every $5 \mathrm{~s}$ in synchronization with the obtained velocity profiles.

\section{RESULTS AND DISCUSSION}

Chemical Shift Artifact during Velocimetric Measurements. Figure 2 shows the ${ }^{1} \mathrm{H}$ NMR spectrum (a) and intensity $I(r)$ (b) and velocity $v(r)$ (c) profiles of olive oil obtained for a $1 \mathrm{~mm}$ gap rheo-microMRI CC cell at constant rotational velocity of $\Omega=0.96 \mathrm{rpm}$. The intensity and velocity profiles clearly show a chemical shift (CS) artifact. Such artifacts are well known to occur for systems where protons have different resonance frequencies due to their different chemical environments and have been discussed thoroughly in the literature. ${ }^{27,38-41}$ For the shown ${ }^{1} \mathrm{H}$ NMR spectrum, we can indeed distinguish different proton signals with a maximal CS difference of $1200 \mathrm{~Hz}$ (4 ppm) (Figure 2(a)). We observed a CS displacement of 25 pixels at the chosen receiver bandwidth of $\mathrm{BW}=25 \mathrm{kHz}$ and matrix size of $N=512$.
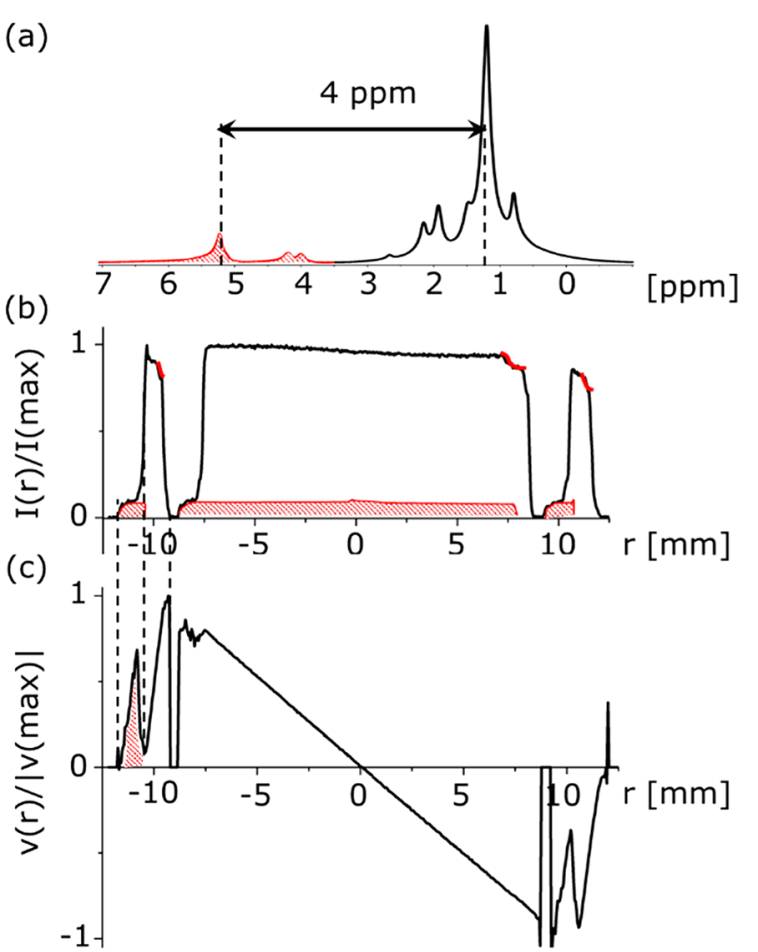

Figure 2. ${ }^{1} \mathrm{H}$ NMR spectrum and chemical shift artifacts observed during rheo-microMRI measurements of olive oil at a magnetic field strength of $\mathrm{B}_{0}$ of $7 \mathrm{~T}$. In the ${ }^{1} \mathrm{H}$ NMR spectrum, (a) the maximal chemical shift difference of $4 \mathrm{ppm}$ is indicated by the black arrow. The indicated chemical shift caused artifacts in normalized intensity $I(r)$ (b) and velocity $v(r)$ (c) profiles. These appear as additional signals, shifted to the right part, of the profiles (black dotted lines are intended to guide the eye). The intensity $I(r)(\mathrm{b})$ and velocity $v(r)$ (c) profiles were obtained as a function of position $r$ in a $1 \mathrm{~mm}$ gap CC rheo-microMRI cell at a constant rotational velocity of $\Omega=0.96$ $\mathrm{rpm}$. The profiles were measured with a pulsed gradient spin echo (PGSE) sequence with $\Delta=15 \mathrm{~ms}$ and a receiver BW of $25 \mathrm{kHz}$.

(Figure 2(b) and (c)). The observed CS artifact in the intensity and velocity profiles is the result of spatial mismapping of the MR signal based on its spectroscopic frequency, which typically will be seen in the read direction $\left(G_{\text {Read }}\right)$ (Figure 1$)$. This mismapping can be reduced by increasing the receiver bandwidth (BW), which results in an increased spectral width per pixel for a given matrix size. In practice, the increase in BW has to be accounted for by an increased read gradient $\left(G_{\text {Read }}\right)$ to obtain the same field of view (FOV) and spatial resolution. Figure 3 demonstrates how the CS artifact, clearly observed in rheo-microMRI intensity and velocity profiles at a BW of $25 \mathrm{kHz}$, was improved by increasing the BW up to $125 \mathrm{kHz}$ (the full set of the rheo-MRI data as a function of the BW can be found in Figure S1). Even though the broadening of the profiles decreased significantly and the deviation from the expected position became less, the CS artifact is still noticeable (about 5 pixels). A further increase in $\mathrm{BW}$ values up to $250 \mathrm{kHz}$ reduced the shift to 3 pixels (Figure S1).

The chemical shift artifact can also be minimized by selecting the proton signal of interest or by suppressing unwanted proton signals. For our experimental setup, we found that the suppression of unwanted peaks was a more efficient approach than selective excitation (Figure S2). To implement the chemical shift suppression approach, we added a CHESS $^{39,42,43}$ module before the flow encoding a pulsed 
(a)

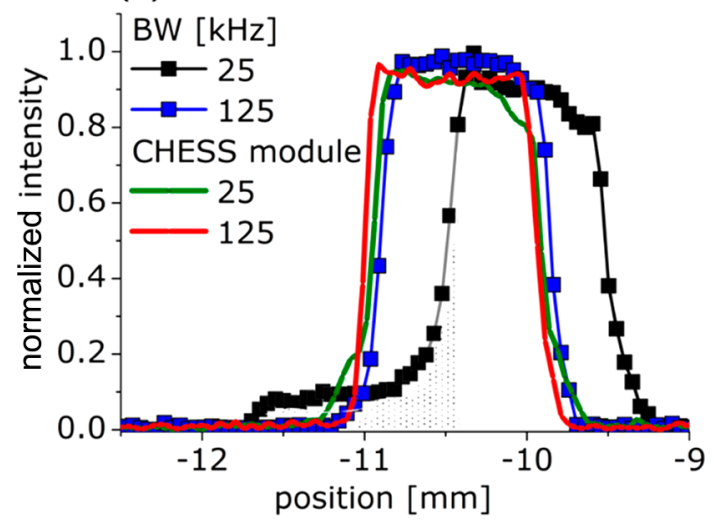

(b)

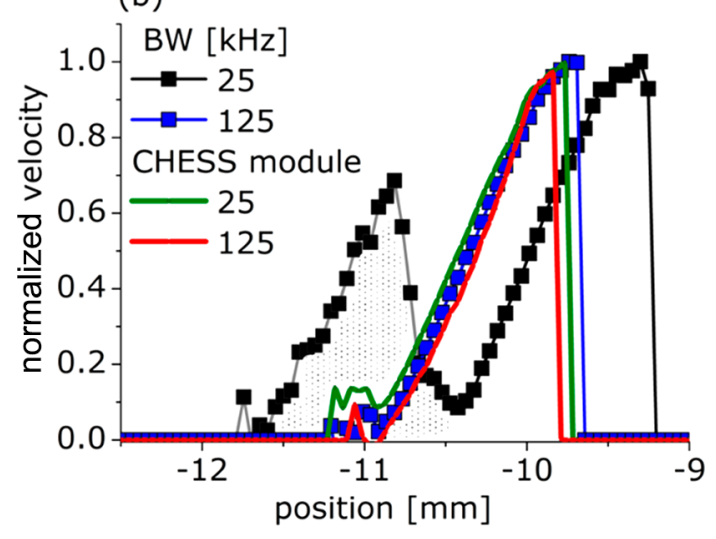

Figure 3. Example of a chemical shift artifact and its minimization by increasing the receiver bandwidth (BW) and use of a chemical shift suppression CHESS module: zoomed in normalized intensity (a) and velocity (b) profiles of olive oil obtained at $B_{0}=7 \mathrm{~T}$ as a function of BW when using a pulsed gradient spin echo (PGSE) sequence (squares) and one modified with a CHESS selection module (lines). All profiles were obtained at a rotational velocity of $\Omega=0.96 \mathrm{rpm}$ in a $1 \mathrm{~mm}$ gap CC rheo-microMRI cell.

gradient spin echo (PGSE) sequence (Figure S3). Three CHESS pulses with frequency selective $90^{\circ} \mathrm{rf}$ pulses for unwanted proton frequencies in combination with spoiler gradients effectively suppress signals from protons different from the on-resonance one that should remain. For olive oil, we applied $90^{\circ}$ pulses with a bandwidth of $1000 \mathrm{~Hz}$ on resonance for protons at a chemical shift difference of $4.2 \mathrm{ppm}$ (Figure 2(a)), suppressing signals between 2.5 and $5.7 \mathrm{ppm}$. The resulting velocity and density profiles are shown in Figure 3. Significantly improved profiles were obtained even for a BW of $25 \mathrm{kHz}$. However, a chemical shift displacement of 6 pixels was still observed in the acquired profiles, which were attributed to protons being $1 \mathrm{ppm}$ off-resonance and therefore not suppressed. The chemical shift artifact still appeared on the edges of the intensity and velocity profiles and should not be understood as wall slip. Hence, we deployed the CHESS suppression pulses in combination with an increased BW (Figure S1). At a BW of $125 \mathrm{kHz}$, we observed well-defined rectangular intensity and straight velocity profiles (Figure 3, red line). The broadening due to CS dispersion now hardly affected the rheo-microMRI measurements.

We note that in previous work we used a different strategy by combining the PGSE with a chemical shift suppression module consisting of a series of frequency-selective $90^{\circ}$ pulses with a time separation of $5 \times \mathrm{T} 2$ between them. ${ }^{44}$ In hindsight, we found no clear advantage to the simpler CHESS suppression approach. Here, we have experimentally demonstrated that a conventional CHESS module provides an effective alternative for reducing chemical shift artifacts.

Artifacts Occurring during Velocimetric Measurements at Low Rotational Velocities. Velocimetric measurements at low rotational velocities $\Omega$, as implemented on our commercial rheo-microMRI setup, required us to work at the edge of possible experimental settings for observation time $\Delta$, flow encoding $G_{\text {Flow, }}$ and flow compensated $G_{\mathrm{FC}}$ gradients (Figure S3). These conditions resulted in velocity profiles with artifacts that were enhanced at short flow encoding time (small $\Delta$ ) and large gradient values, which were intrinsic to the pulse sequence. We distinguished artifacts related to gradient imperfections and to mechanical instabilities of the used rheo-microMRI setup.

The gradient-related artifacts appeared in noncentered and nonsymmetric velocity profiles with negative velocities on one side and unrealistically high velocities on another side (Figure S4). The generated artifacts can be seen more clearly if we perform velocimetric measurements at zero rotational velocity (Figure S5). This artifact does not relate to the flow itself and shows a first-order linear phase shift which can be corrected for. The shift of the velocity data is likely to be caused by a phase shift related to technical imperfections of the gradient systems such as eddy currents. ${ }^{40,45}$ One route to minimize the influence of these artifacts is to record the velocities at reduced $G_{\text {Flow }}$ gradient strength. For our commercial rheo-microMRI setup and its related sequences to measure flow, however, the implementation of $G_{\mathrm{FC}}$ gradients along the read direction (Figure S3) forced us to use a short observation time $(\Delta)$ leading to strong $G_{\text {Flow }}$ gradients to record low velocities. ${ }^{19}$ Once the $G_{\mathrm{FC}}$ gradients were disabled, by modifying the pulse sequence, a longer $\Delta$ could be used, which resulted in a reduction in $G_{\text {Flow }}$ and an increase in sensitivity of the velocimetric measurements at low rotational velocities $\Omega$.

As a workaround for the non-flow related phase shifts, a correction of the (phase-shifted) noncentered velocity profile was used in postprocessing. This approach is based on the expected velocity profile for the rotating inner cylinder of the rheo-MRI CC cell, which is supposed to be centered and linear as a function of position $r$ (Figure S4). First, a theoretical velocity profile was calculated based on the actual applied velocities and was centered, considering the symmetry of the CC cell. Next, the measured velocities of the inner cylinder were analyzed by a linear fit. The fitted and theoretical velocity profiles for the rotating inner cylinder were extrapolated over the whole dimension of the rheo-cell (FOV $25 \mathrm{~mm}$ ). The difference between them was used to correct the initially measured velocity profile, including velocities within the gaps. The correction resulted in well-centered and symmetric velocity profiles (Figure S4).

The above-mentioned remedies also alleviated the impact of mechanical instabilities due to wobbling of the rotating inner cylinder of the CC cell (Figure S6(a)). Reducing the gradient strengths of $G_{\text {Flow }}$ by increasing the observation time $\Delta$ effectively reduced the impact of wobbling on the velocity profile (Figure S6(b). This approach turned out to be more effective in suppressing the wobbling artifact and less time consuming than using a trigger to record velocity profiles in sync with the rotation of the inner cylinder (Figure S6(c)). 
Recently, the effects of curved streamlines in relation to slice thicknesses as they occur in millimeter-sized gaps of CC cells on the accuracy of the velocity profiles have been discussed, ${ }^{20,34}$ and the chosen slice thickness of $1-2 \mathrm{~mm}$ was in line with the recommendations that were made. Therefore, in our current work, we can assume that the impact of curved stream lines was minor.

Local Flow Curves: Validation for Wide Gap CC (Couette) Geometry. The previously described approaches to obtain artifact free rheo-MRI velocity profiles for fluids in CC cells at high magnetic field strength opened up the possibility to obtain LFCs in a quantitative manner. ${ }^{30}$ First, shear rate profiles $\dot{\gamma}(r)$ were deduced from rheo-MRI velocity profiles according to the equation $\dot{\gamma}(r)=r \partial(v(r) / r) / \partial r$ (Figure 1(c)). Macroscopic torque $T$ values were obtained with an identical CC cell mounted in a conventional rotational rheometer and allowed for the calculation of shear stress as a function of position using $\sigma(r)=T / 2 \pi \mathrm{Hr}^{2}$ (Figure 1(c)), where $H$ is the fluid height in the gap, and $r$ is the position within the gap. This equation is obtained from the momentum equation assuming the absence of significant normal stress differences, edge effects, and fluid inertia. ${ }^{6,28}$ We used different gap sizes to vary the shear stress field which is a function of the gap size and position as in $\sigma(r)=\sigma_{\mathrm{i}} r_{\mathrm{i}}^{2} / r^{2}$, where $\sigma_{\mathrm{i}}$ is the stress exerted by the rotating inner cylinder, and $r_{\mathrm{i}}$ the radius of the inner cylinder. By using rheo-microMRI CC cells with gap sizes of $1,2.5$, and $4 \mathrm{~mm}$, shear stress variations of $17 \%, 40 \%$, and $60 \%$ could be applied, respectively.

This approach was validated by a comparison of LFCs with global flow curves for silicone oil and a commercial Carbopol dispersion (hair gel) as examples of Newtonian and yield stress fluids, respectively. These model fluids were selected since their flow behavior is neither heterogeneous nor time dependent. That made them well suited to validate the approach to obtain quantitative LFCs, since these should be identical to the global flow curves obtained by a conventional rotational rheometer.

Figure 4(a) shows global flow curves for silicone oil, displaying the expected linear behavior for a Newtonian fluid. The LFCs measured for silicone oil in CC cells all overlap with the global flow curves for the applied gap sizes $(1,2.5$, and 4 $\mathrm{mm})$ and the applied rotational velocities $\Omega$ between 1 and 32 rpm. We conducted a two-way ANOVA test to evaluate the effects of the method used (GFC vs LFC) and the gap with (1, 2.5 , and $4 \mathrm{~mm}$ ) on the estimated viscosity values of silicone oil. There was no significant effect of the applied method and gap width on viscosity (at $\alpha=0.05$, Table S1). The precisions of the two methods were however different, based on 95\% confidence intervals of the means (viscosities obtained by GFC and LFC were, respectively, $0.512 \pm 0.007$ and $0.562 \pm 0.069$ ). Based on this two-way ANOVA test, we concluded that the estimated viscosities obtained by GFC and LFC were not statistically different from each other. These methods only differed with respect to measurement precision. The high precision of the viscosity as determined by GFC is in line with the experimentally determined relative standard deviation of $1 \%$ for silicone oil on this rheometer, as verified by the supplier of the instrument. We deem the lower precision of the viscosities obtained from LFCs acceptable for practical applications. In our second ANOVA test, we tested LFCs for the influence of gap width $(2.5,4.0 \mathrm{~mm})$ and rotation velocity $(\Omega=2,4,8,16,32 \mathrm{rpm})$ on the estimated viscosity of the silicone oil. ANOVA showed there was no significant effect of
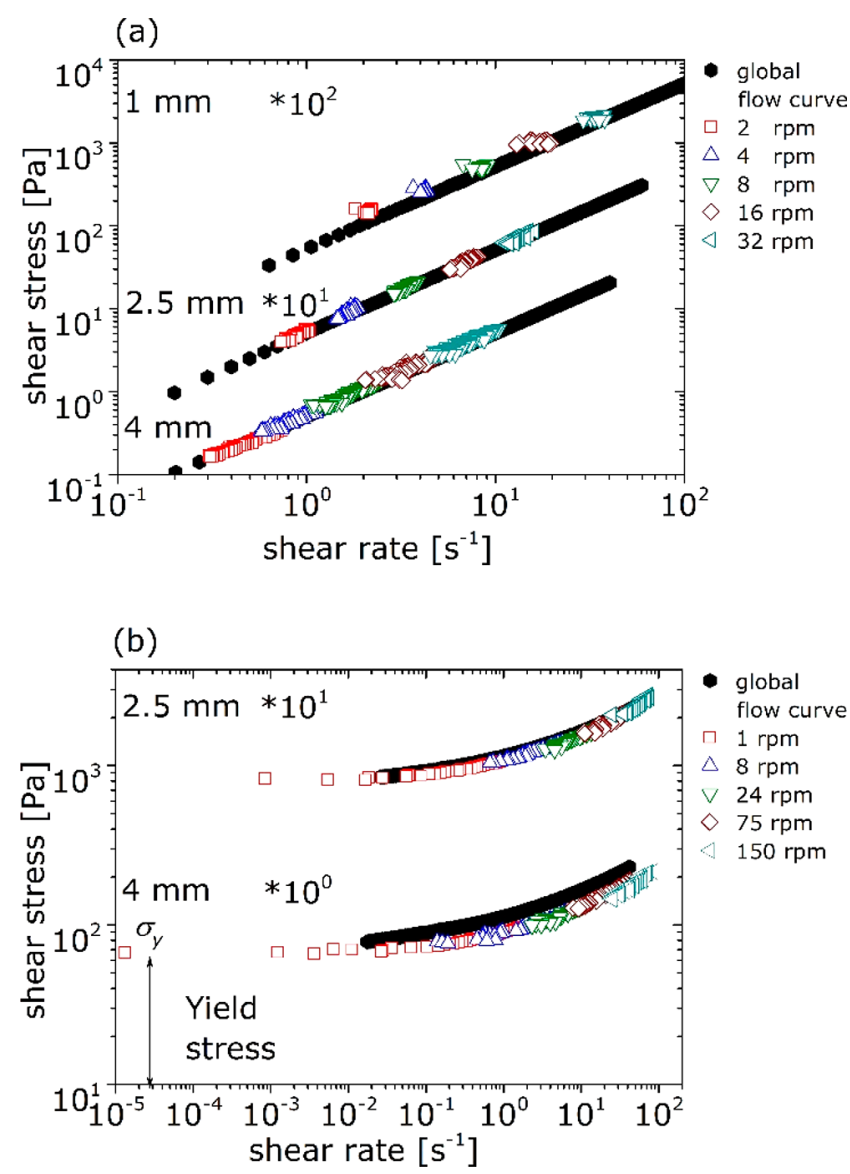

Figure 4. Local and global flow curves obtained for silicone oil (a) and hair gel (b), respectively, representing Newtonian and yield stress fluids. The local flow curves (LFC) $\sigma(\dot{\gamma}(\mathrm{r}))$ were obtained with applied rotational velocities $\Omega$ displayed in the legend. To aid the visualization, the local and global flow curves obtained in CC cells with different gap sizes $(1,2.5$, and $4 \mathrm{~mm})$ are presented with an offset (multiplication factors are indicated in the plot).

gap width and rotation speed on viscosities obtained by LFCs. No systematic effect of gap width and $\Omega$ was present, as these were estimated as random factors in the ANOVA analysis (Tables S1 and S2). This demonstrates that LFCs can be obtained in a quantitative manner for practically relevant gap sizes and shear rate conditions. This agreement was also observed for the hair gel (Figure 4(b)), where the applied rotational velocities $\Omega$ were varied between 1 and $150 \mathrm{rpm}$. For this Carbopol dispersion, the global flow curve can be well fitted with a single power law function and be described with Herschel-Bulkley behavior, as expected for a simple yield stress fluid. ${ }^{8,46,47}$ The good match between the GFC and respective LFCs in Figure 4 is reflected in the small $(<10 \%)$ variation of the yield stress obtained by the Herschel-Bulkley model for these two methods, as well as gap width and $\Omega$ (Table S3). This validates that quantification of complex material properties can be performed via rheo-microMRI.

Local Flow Curves: A Local View on Transient Structure Formation and Degradation. The potential of real-time assessment of LFCs by rheo-microMRI was demonstrated for a dispersion of micronized fat crystals (MFCs) in sunflower oil, which is known to form a network under low shear ${ }^{15,48}$ (Figure 5). The MFC dispersion was measured in a $2.5 \mathrm{~mm}$ gap-sized rheo-MRI CC cell at a 
(a)

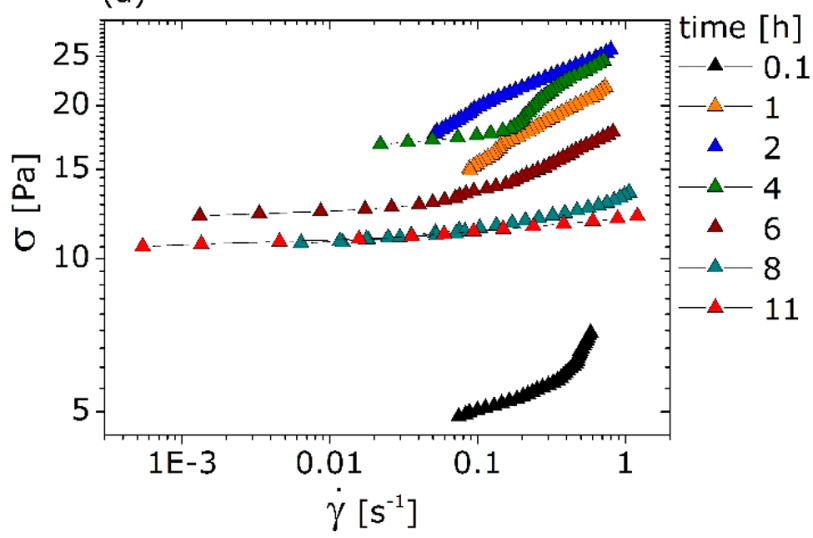

(b)
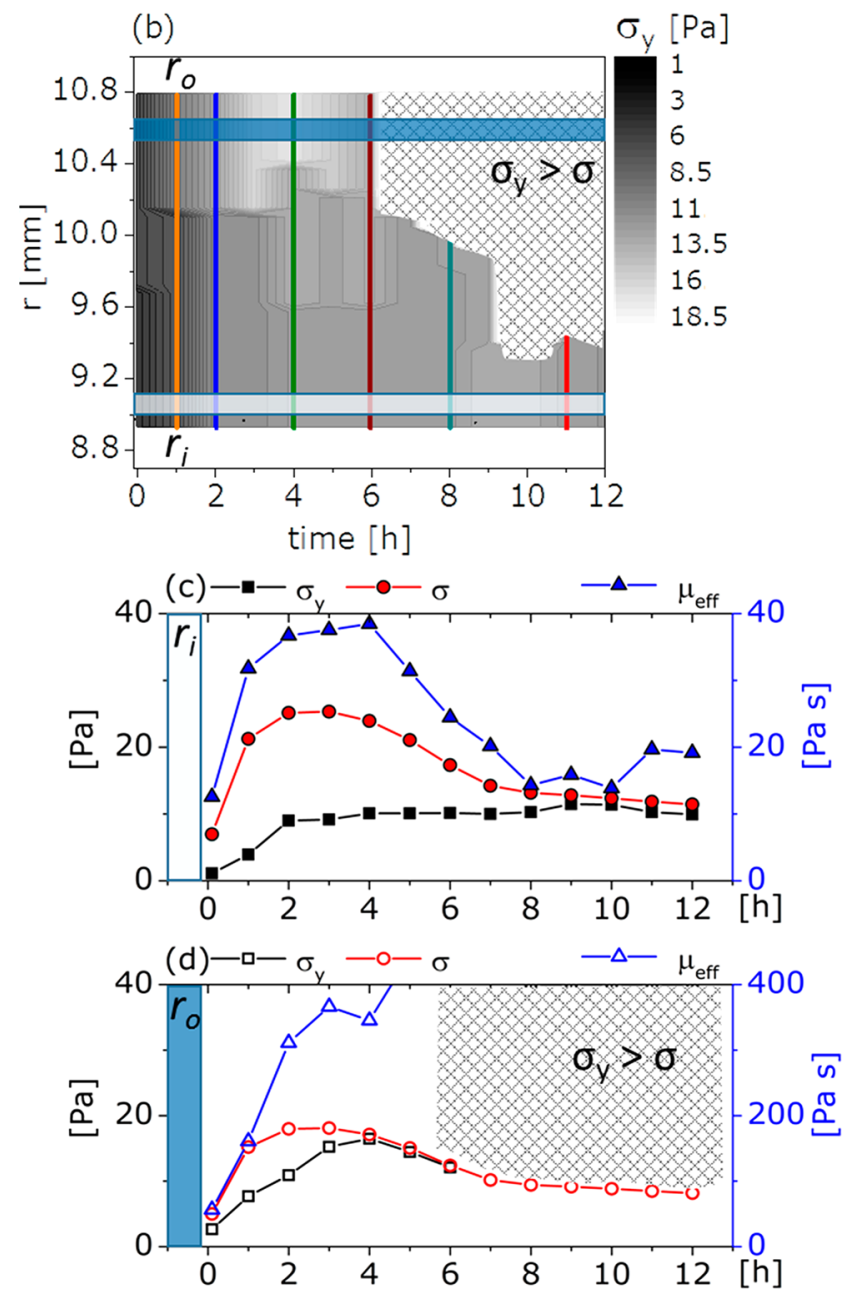

Figure 5. (a) Evolution of local flow curve (LFC) $\sigma(\dot{\gamma}(r))$ reflecting network formation of micronized fat crystals in sunflower oil under shear stress at a rotational velocity of $\Omega=0.96 \mathrm{rpm}$ in a rheomicroMRI CC cell with a $2.5 \mathrm{~mm}$ gap size. (b) $2 \mathrm{D}$ map of the yield stress $\sigma_{y}$, resulting from a power law fit, as a function of position within the gap and time. The hatched area indicates regions with no flow. Colored lines indicate how regions in panel (b) corresponded to LFCs in panel (a). For two positions, indicated with light and dark blue columns, the local yield stress and effective viscosity $\mu_{\text {eff }}$ were plotted as a function of time in respectively (c) and (d).

rotational velocity of $\Omega=0.96 \mathrm{rpm}$ for $13 \mathrm{~h}$. The velocity profiles, recorded every $5 \mathrm{~min}$, showed a continuous development of heterogeneous flow behavior within the gap during whole experiment (Figure S7). In parallel, the same dispersion was placed into an identical CC cell mounted in a commercial rheometer to measure $T$ values over the same time frame, imposed at constant rotational velocity $\Omega$. The evolution of torque $T$ demonstrated three stages: an increase during the first $3 \mathrm{~h}$, a fast decrease within the next $4 \mathrm{~h}$, and a subsequent slow decrease (Figure S8). This pattern could be interpreted as initial formation of the MFC network followed by subsequent disruption. ${ }^{15,48}$ The corresponding timedependent LFCs are shown in Figure 5(a), and they point to the development of a yield stress fluid. The LFCs could not be fitted with a single Herschel-Bulkley model over the whole range of shear rates for most of the time points, which indicates that the MFC dispersion at different positions in the CC gap was governed by different constitutive laws. The LFCs, however, could be described by a two (or even three) component Herschel-Bulkley models, correlated to different bands in the CC gap (Figure S9). To find an interpretation for the multicomponent LFCs, the different regions were then fitted with a power law function representing the HerschelBulkley model. In this way, local yield stress values $\sigma_{\mathrm{y}}$ over the gap were estimated. The results are presented as a function of time in the two-dimensional map in Figure 5(b). Initially, for all positions over the gap, an overall increase in apparent yield stress $\sigma_{\mathrm{y}}$ could be observed, indicating formation of a MFC network. The map also shows that this increase in yield stress was heterogeneous over the gap. For two positions at different distances from the rotating inner cylinder $r_{\mathrm{i}}$ (light and dark blue columns indicate these positions in Figure 5(b)), we have plotted the local time dependences of $\sigma_{\mathrm{y}}$ (Figure 5(c) and (d)). One can observe that for the position close to the inner rotating cylinder the apparent yield stress $\sigma_{\mathrm{y}}$ was always overcome by a larger local shear stress $\sigma$ (Figure 5(c)). Further away from the rotating cylinder, the apparent yield stress $\sigma_{\mathrm{y}}$ became too high to be overcome by the local stress after $5-6 \mathrm{~h}$ (Figure 5(d)). Hence, after this period, this region became stagnant (hatched area in Figure 5(b)). In Figure 5(c) and (d), we have also presented the local effective viscosity $\mu_{\text {eff }}(r)=$ $\sigma(r) / \dot{\gamma}(r)$. In the region near the rotating inner wall, we observed an increase in $\mu_{\text {effi }}$ followed by a decrease. This was similar to our previous results obtained in a $1 \mathrm{~mm}$ gap with a much more homogeneous stress distribution, where it was concluded that the rapid increase in the apparent $\sigma_{y}$ corresponded to the formation of a weak-link MFC network, ${ }^{15}$ followed by a decrease corresponding to its disruption due to recrystallization and aggregation of the MFCs. ${ }^{48}$ In the wider CC gap, a static band appeared in time in the region near the outer wall (Figure 5(d)), where the MFC network can continue to grow in strength. These results illustrate the potential of rheo-microMRI for assessment of time-dependent and spatially heterogeneous constitutive laws. Reproducibile network formation in CC cells was observed for dispersions of MFCs in sunflower and bean oils measured in $1,2.5$, and $4 \mathrm{~mm}$ gap-sized rheo-MRI CC cells at rotational velocities of 0.96 and $3 \mathrm{rpm}$.

We note that in our approach we did not measure the macroscopic torque $T$ in situ in a CC cell mounted in a MRI probehead. Current designs for in situ torque measurement in a CC cell in a superconducting magnet are compromised by the long shaft and consequent mechanical instabilities, ${ }^{49}$ which limits the operational range of $\Omega$ and $T$. Our approach employing two parallel measurements delivered reproducible 
results for a wide range of rotational velocities $(\Omega=0.1-150$ $\mathrm{rpm})$ and torques $(T=0.01-100 \mathrm{mN} \mathrm{m})$ and can be implemented in a straightforward manner in laboratories equipped with a standard wide bore, high field, NMR spectrometer, equipped with a microMRI probehead and a commercial rotational rheometer using the same rheo cell.

\section{CONCLUSIONS}

Chemical shift artifacts in rheo-microMRI at a high $\mathrm{B}_{0}$ field can be minimized by introducing CHESS pulses to suppress unwanted proton signals. By also accounting for eddy currents generated by pulsed field gradients, velocity profiles can be obtained that are virtually free of artifacts. This allows for construction of LFCs which quantitatively match global flow curves for Newtonian and simple yield stress fluids. No systematic effects of gap size and rotational velocities on the viscosity of a Newtonian fluid and yield stress of a complex fluid could be observed. The approach allows for assessment of the transient local constitutive laws for micronized fat crystal dispersion that undergo structural rearrangements in a CC cell under shear stress.

\section{ASSOCIATED CONTENT}

\section{(s) Supporting Information}

The Supporting Information is available free of charge at https://pubs.acs.org/doi/10.1021/acs.analchem.9b03216.

Intensity and velocity profiles demonstrating chemical shift and wobbling artifacts and their minimization and numerical data of velocity, shear rate, and torque values to reconstruct local flow curves and their fitting by a power law function (PDF)

\section{AUTHOR INFORMATION}

\section{Corresponding Author}

John van Duynhoven - Laboratory of Biophysics, Wageningen University \& Research, 6708 WE Wageningen, The Netherlands; Unilever Food Innovation Centre, 6708 WH Wageningen, The Netherlands; MAGNEFY, 6708 WE Wageningen, The Netherlands; 10 orcid.org/0000-0001-97690113; Phone: +31317482044; Email: john.vanduynhoven@ wur.nl

\section{Authors}

Tatiana Nikolaeva - Laboratory of Biophysics, Wageningen University \& Research, 6708 WE Wageningen, The Netherlands; MAGNEFY, 6708 WE Wageningen, The Netherlands; O orcid.org/0000-0003-2029-0060

Frank J. Vergeldt - Laboratory of Biophysics, Wageningen University \& Research, 6708 WE Wageningen, The Netherlands; MAGNEFY, 6708 WE Wageningen, The Netherlands

Raquel Serial - Laboratory of Biophysics, Wageningen University \& Research, 6708 WE Wageningen, The Netherlands; MAGNEFY, 6708 WE Wageningen, The Netherlands

Joshua A. Dijksman - Physical Chemistry and Soft Matter, Wageningen University \& Research, 6708 WE Wageningen, The Netherlands; (1) orcid.org/0000-0002-8337-1434

Paul Venema - Physics and Physical Chemistry of Foods, Wageningen University \& Research, 6708 WG Wageningen, The Netherlands
Adrian Voda - Unilever Food Innovation Centre, 6708 WH Wageningen, The Netherlands; 10 orcid.org/0000-0002-63407903

Henk Van As - Laboratory of Biophysics, Wageningen University \& Research, 6708 WE Wageningen, The Netherlands; MAGNEFY, 6708 WE Wageningen, The Netherlands

Complete contact information is available at:

https://pubs.acs.org/10.1021/acs.analchem.9b03216

\section{Author Contributions}

T.N., H.V.A., and J.v.D. designed the research. F.J.V. assisted in the selection and optimization of the MRI acquisition and data processing. T.N. performed the MRI and rheological experiments. R.S. assisted in the work to reduce the chemical shift artifact. J.A.D. and P.V. assisted in interpreting the rheological results. A.V. selected the model dispersion and the interpreted results. All authors have given approval to the final version of the manuscript.

\section{Notes}

The authors declare the following competing financial interest(s): J.v.D. and A.V. are employed by a company that manufactures lipid-based food products.

\section{ACKNOWLEDGMENTS}

John Philippi and the technical workshop of Wageningen University are thanked for designing and manufacturing the CC cells. Daan de Kort is acknowledged for his input on the design and fruitful discussion of the first rheological and rheoMRI experiments. Julia Krug is acknowledged for input on the design of the MRI experiments. Ewoud van Velzen (Unilever $\mathrm{R} \& \mathrm{D}$, Wageningen) provided expert advice on ANOVA. This work is part of the SSCANFOODS (project number 13386) research program, which is financed by The Netherlands Organisation for Scientific Research (NWO).

\section{REFERENCES}

(1) Balmforth, N. J.; Frigaard, I. A.; Ovarlez, G. Annu. Rev. Fluid Mech. 2014, 46 (1), 121-146.

(2) Coussot, P. J. Non-Newtonian Fluid Mech. 2014, 211, 31-49.

(3) Macias-Rodriguez, B. A.; Marangoni, A. A. Crit. Rev. Food Sci. Nutr. 2018, 58, 2398-2415.

(4) Mezger, T. G. The Rheology Handbook, 4th ed.; Vincentz Network: Hannover, Germany, 2012. DOI: 10.1515/ 9783748600367.

(5) Macosko, C. W. Rheology: Principles, Measurements and Applications; Wiley-VHC, 1996; Vol. 86.

(6) de Kort, D. W.; Nikolaeva, T.; Dijksman, J. A. Rheo-NMR: Applications to Food. In Modern Magnetic Resonance.; Webb, G. A., Ed.; Springer, Cham, 2017; pp 1-21. DOI: 10.1007/978-3-31928275-6 19-1.

(7) Dekkker, R. I.; Dinkgreve, M.; de Cagny, H.; Koeze, D. J.; Tighe, B. P.; Bonn, D. J. Non-Newtonian Fluid Mech. 2018, 261, 33-37.

(8) Möller, P.; Fall, A.; Chikkadi, V.; Derks, D.; Bonn, D. Philos. Trans. R. Soc., A 2009, 367 (1909), 5139-5155.

(9) Coussot, P.; Nguyen, Q. D.; Huynh, H. T.; Bonn, D. J. Rheol. 2002, 46 (3), 573.

(10) Fall, A.; Lemaître, A.; Ovarlez, G. EPJ Web Conf. 2017, 140, 09001.

(11) Callaghan, P. T.; Rogers, S. A.; Vlassopoulos, D. Phys. Rev. Lett. 2008, 100, 128304.

(12) Ragouilliaux, A.; Herzhaft, B.; Bertrand, F.; Coussot, P. Rheol. Acta 2006, 46, 261-271.

(13) Masselon, C.; Salmon, J.-B.; Colin, A. Phys. Rev. Lett. 2008, 100, 38301. 
(14) Goyon, J.; Colin, A.; Ovarlez, G.; Ajdari, A.; Bocquet, L. Nature 2008, 454, 84.

(15) Nikolaeva, T.; den Adel, R.; Velichko, E.; Bouwman, W. G.; Hermida-Merino, D.; Van As, H.; Voda, A.; van Duynhoven, J. Food Funct. 2018, 9, 2102-2111.

(16) Rodts, S.; Boujlel, J.; Rabideau, B.; Ovarlez, G.; Roussel, N.; Moucheront, P.; Lanos, C.; Bertrand, F.; Coussot, P. Phys. Rev. E Stat. Nonlinear, Soft Matter Phys. 2010, 81, 021402.

(17) Ovarlez, G.; Bertrand, F.; Rodts, S. J. Rheol. 2006, 50 (3), 259292.

(18) Britton, M. M.; Callaghan, P. T. J. Texture Stud. 2000, 31 (3), 245-255.

(19) Callaghan, P. T. Translational Dynamics and Magnetic Resonance: Principles of Pulsed Gradient Spin Echo NMR; Oxford University Press: Oxford, 2011. DOI: 10.1093/acprof:oso/ 9780199556984.001.0001.

(20) Kuczera, S.; Galvosas, P. J. Magn. Reson. 2015, 259, 135-145.

(21) Galvosas, P.; Brox, T.; Kuczera, S. Magn. Reson. Chem. 2019, 57

(9), 757-765.

(22) Callaghan, P. T. Rheol. Acta 2008, 47, 243-255.

(23) Al-kaby, R. N.; Jayaratne, J. S.; Brox, T. I.; Codd, S. L.; Seymour, J. D.; Brown, J. R. J. Rheol. 2018, 62 (5), 1125-1134.

(24) Callaghan, P. T. Curr. Opin. Colloid Interface Sci. 2006, 11 (1), 13-18.

(25) Callaghan, P. T. Rep. Prog. Phys. 1999, 62 (4), 599-670.

(26) Callaghan, P. T.; Samulski, E. T. Macromolecules 1997, 30, $113-122$.

(27) Broadbent, A. L.; Mullin, J. M.; Codd, S. L.; Dockery, J. D.; Seymour, J. D. Appl. Magn. Reson. 2012, 42 (1), 137-152.

(28) Bird, R. B. Dynamics of Polymeric Liquids; Wiley: New York, 1987; Vol. 2.

(29) Ovarlez, G.; Rodts, S.; Ragouilliaux, A.; Coussot, P.; Goyon, J.; Colin, A. Phys. Rev. E - Stat. Nonlinear, Soft Matter Phys. 2008, 78, 036307.

(30) Coussot, P.; Tocquer, L.; Lanos, C.; Ovarlez, G. J. NonNewtonian Fluid Mech. 2009, 158 (1-3), 85-90.

(31) Divoux, T.; Fardin, M. A.; Manneville, S.; Lerouge, S. Annu. Rev. Fluid Mech. 2016, 48 (1), 81-103.

(32) Ovarlez, G.; Rodts, S.; Chateau, X.; Coussot, P. Rheol. Acta 2009, 48 (8), 831-844.

(33) Møller, P. C. F.; Rodts, S.; Michels, M. A. J.; Bonn, D. Phys. Rev. E - Stat. Nonlinear, Soft Matter Phys. 2008, 77, 041507.

(34) Colbourne, A. A.; Blythe, T. W.; Barua, R.; Lovett, S.; Mitchell, J.; Sederman, A. J.; Gladden, L. F. J. Magn. Reson. 2018, 286, 30-35. (35) Callaghan, P. T. Rheo-NMR: A New Window on the Rheology of Complex Fluids. In Encyclopedia of Nuclear Magnetic Resonance; Grant, D. M., Harris, R. K., Eds.; John Wiley \& Sons Ltd., 2002; Vol. 9, pp 737-750. DOI: 10.1002/9780470034590.emrstm0470.

(36) Britton, M. M.; Callaghan, P. T.; Kilfoil, M. L.; Mair, R. W.; Owens, K. M. Appl. Magn. Reson. 1998, 15 (3-4), 287-301.

(37) Tayler, A. B.; Benning, M.; Sederman, A. J.; Holland, D. J.; Gladden, L. F. Phys. Rev. E 2014, 89 (6), 063009.

(38) Elkins, C. J.; Alley, M. T. Exp. Fluids 2007, 43 (6), 823-858.

(39) Bley, T. A.; Wieben, O.; Francois, C. J.; Brittain, J. H.; Reeder,

S. B. J. Magn. Reson. Imaging 2010, 31 (1), 4-18.

(40) Middione, M. J.; Thompson, R. B.; Ennis, D. B. Magn. Reson. Med. 2014, 71 (6), 2014-2023.

(41) Brown, J. R. Colloidal Suspension Flow and Transport Behavior in Small Channels by Magnetic Resonance Microscopy. Ph.D. Thesis, Montana State University, 2007.

(42) Lakshmanan, S.; Maru, W. A.; Holland, D. J.; Mantle, M. D.; Sederman, A. J. Flow Meas. Instrum. 2017, 53, 161-171.

(43) Haase, A.; Frahm, J. J. Magn. Reson. 1985, 64 (1), 94-102.

(44) Serial, M. R.; Nikolaeva, T.; Vergeldt, F. J.; van Duynhoven, J.; Van As, H. Magn. Reson. Chem. 2019, 57, 766-770.

(45) Peeters, J. M.; Bos, C.; Bakker, C. J. G. Magn. Reson. Med. 2005, 53 (1), 126-133.

(46) Dinkgreve, M.; Fazilati, M.; Denn, M. M.; Bonn, D. J. Rheol. 2018, 62 (3), 773-780.
(47) Dinkgreve, M.; Denn, M. M.; Bonn, D. Rheol. Acta 2017, 56 (3), 189-194.

(48) Nikolaeva, T.; den Adel, R.; van der Sman, R.; Martens, K. J. A.; Voda, A.; Van As, H.; van Duynhoven, J. Langmuir 2019, 35 (6), 2221-2229.

(49) Brox, T. I.; Douglass, B.; Galvosas, P.; Brown, J. R. J. Rheol. 2016, 60 (5), 973-982. 